\title{
El concepto de pseudoindustrialización de Milcíades Peña en "La evolución industrial argentina" (Revista Fichas, Abril 1964)
}

\author{
Milcíades Peña's concept of pseudoindustrialization in "Argentina's industrial \\ evolution" (Fichas journal, April 1964)
}

José Facundo Manzanelli*

\begin{abstract}
Resumen: En este trabajo nos acercaremos a un concepto que desarrolló Milcíades Peña -intelectual y militante trotskista argentino- con el fin de polemizar con la intelectualidad peronista sobre el proceso de industrialización en Argentina. A partir de la contextualización de la actividad política de Peña en los ' 50 y '60 se analizará el concepto de pseudoindustrialización que el autor desarrolla en "La evolución industrial argentina". Artículo aparecido en el primer número de la revista Fichas de investigación económica y social (Abril 1964), y escrito bajo el pseudónimo de Víctor Testa.
\end{abstract}

Palabras clave: Milcíades Peña - morenismo - peronismo - pseudoindustrializaciónlatifundio - imperialismo.

\begin{abstract}
In this paper, we take a closer look at a concept developed by the Argentinian intellectual and trotskyist militant Milcíades Peña, with the purpose debating with the Peronist intellectuals about the process of industrialization in Argentina. Taking as our starting point Peña's political activity during the 50's and 60 's, the concept of pseudoindustrialization will be analyzed. This concept, developed by the author, initially appeared in the article "La evolución industrial argentina", in the first edition of the magazine Fichas de investigación económica y social (Abril 1964), and was written under the pseudonym Víctor Testa.
\end{abstract}

Key words: Milcíades Peña - Morenism - Peronism - pseudoindustrialization latifundia - imperialism.

\section{Introducción}

En este trabajo nos acercaremos a un concepto que desarrolló un importante intelectual de la historiografía marxista argentina quien fuera también militante de la tradición trotskista: Milcíades Peña (1933-1965). Específicamente se tratará el concepto de

\footnotetext{
*Argentino, Profesor en Historia por la Universidad Nacional de Córdoba (Argentina). Facu_cat@ hotmail.es
} 
pseudoindustrialización que el autor desarrolla en "La evolución industrial argentina". Artículo aparecido en el primer número de la revista Fichas de investigación económica y social (abril 1964), y escrito por el mismo bajo el pseudónimo de Víctor Testa.

Para ello, en primera instancia, abordaremos la historia del trotskismo argentino de mediados del siglo pasado para dar un marco a la tarea intelectual de Peña. Se tendrán en cuenta sus vínculos con el morenismo y los debates que suscitaron cuestiones como el peronismo, la industrialización argentina, la revolución socialista en países atrasados, y el rol de la clase obrera argentina. Autores como Horacio Tarcus, Hernán Camarero y Osvaldo Coggiola, desde distintas posiciones y ángulos, brindan opciones para realizar esta contextualización de la acción política e intelectual del autor en cuestión.

Luego pasaremos al abordaje del concepto propiamente dicho. Cuáles son los componentes teóricos de la pseudoindustrialización que caracterizan la realidad argentina en los años peronistas, y cuáles son las implicancias políticas de estos planteos. Metodológicamente se descompondrá el análisis en distintos planos: la inserción del país en el mercado mundial, las relaciones de propiedad de los medios de producción y, por último, las relaciones entre las distintas clases sociales. Veremos aquí como funciona en Peña la tradición marxista heredada desde los aportes teóricos y metodológicos de Lenin y Trotsky, y cómo son útiles a las polémicas que lleva a cabo el autor con los apologistas de la industrialización peronista durante la década del ' 60 .

Por último consideraremos el aporte de las críticas de Osvaldo Coggiola para explicar los errores y aciertos del planteo teórico de Milcíades Peña en los planos intelectual y político.

\section{Milcíades Peña y el trotskismo en la argentina peronista}

Para exponer sobre el trabajo de Peña en la revista Fichas es importante primero introducir la situación política en la que se inserta el autor. Podemos plantear que su formación intelectual y militante corrió por cuenta del trotskismo argentino bajo el signo de Nahuel Moreno (pseudónimo de Hugo Miguel Bressano) en dos grandes etapas.

La primera etapa corresponde al ingreso a la política partidaria a fines de los años ' 40 buscando introducirse en el marxismo siendo apenas un adolescente que, en un contexto de crisis del Partido Socialista, se refugia en la alternativa trotskista ${ }^{1}$. Peña ingresa al Grupo Obrero Marxista (GOM) que tenía su origen en una escisión del Partido Obrero Revolucionario Socialista (PORS). En los primeros años '40 las divisiones del trotskismo se van a acentuar por un fenómeno que irrumpirá en la política y sociedad argentina, y que atravesará el pensamiento, las estrategias y la organización partidaria de la izquierda marxista: el peronismo ${ }^{2}$.

Las caracterizaciones del peronismo que el GOM-POR va a realizar influyeron en las consideraciones de Peña acerca del mismo. Las cambiantes posiciones de la organización fueron justificadas teórica y políticamente mientras impregnaban los juicios teóricos de Peña hacia el peronismo en los años '40 y '50.

\footnotetext{
${ }^{1}$ Camarero Hernán, "El período formativo de un intelectual: Milcíades Peña y el trotskismo en las décadas de 1940-1950", Archivos de Historia del movimiento obrero y la izquierda, $\mathrm{n}^{\circ}$ 3, septiembre 2013, pág. 11.

${ }^{2}$ Coggiola Osvaldo, Historia del trotskismo en Argentina y América Latina, Buenos Aires, Ediciones ryr, 2006, pág. 103.
} 
Luego del 17 de octubre de 1945, y al momento que Perón gana las elecciones en el '46, el GOM, señala Coggiola, tenía una posición sectaria frente al peronismo ${ }^{3}$. Para Moreno, y para Peña -que en ese momento colaboraba con las investigaciones teóricas del grupo trotskista- el peronismo era una expresión más de los sectores militares y golpistas de Argentina. El gobierno de Perón era de carácter bonapartista con rasgos fascistas, agente del imperialismo británico, con la estructura sindical burocratizada y dirigida como apéndice estatal desde la $\mathrm{CGT}^{4}$. Frente al peronismo la clase obrera aparecía como actor heterónomo y sin conciencia revolucionaria. El 17 de Octubre había sido llevada de las narices por su líder y por la burocracia cegetista. Frente a esto la política de la organización debía orientarse a ganar espacios en los sindicatos para "desperonizarlos" y a la vez lograr "proletarizar al partido".

Desde 1948 el GOM pasa a llamarse Partido Obrero Revolucionario (POR) por el crecimiento en el ámbito sindical y luego, en 1953, integra el Partidos Socialista de la Revolución Nacional (PSRN) dirigiendo la Federación Bonaerense. Con esta primera manifestación de política "entrista", el partido da un giro en su consideración respecto al peronismo. Debía mantener oposición frente al mismo, pero ahora en contra de la creciente presencia imperialista norteamericana, de los sectores reaccionarios del Ejército y de la Iglesia $^{5}$. Estos sectores derrocarán a Perón en 1955 y a partir de allí se hará manifiesta la necesidad de entrar en el peronismo, es decir, de llevar a cabo una política "entrista" al movimiento peronista para lograr hegemonía dentro de un Frente Nacional, lograr la legalidad del peronismo y traer de vuelta a Perón del exilio ${ }^{6}$. Para Coggiola esto es una manifestación de adaptación oportunista ${ }^{7}$.

En el marco de la Revolución Libertadora el morenismo creará el fugaz Movimiento de Agrupaciones Obreras (1956-57), y luego editará Palabra Obrera (PO) donde participa Milcíades Peña -bajo el pseudónimo de Hermes Radio- defendiendo la política de confluencia de las organizaciones dentro del peronismo. Para 1964 la sumisión de la política clasista e independiente ante el "entrismo" en el peronismo llegó al extremo de presentar un resultado desgastante en la militancia, con divisiones internas, oportunismo en la política y pérdida de identidad teórica de $\mathrm{PO}^{8}$.

Osvaldo Coggiola explica estas oscilaciones entre el sectarismo o la capitulación oportunista del trotskismo argentino (algo compartido por las agrupaciones de esta línea) de segunda mitad de la década del '50, en el marco de la incapacidad que tuvieron las agrupaciones para "ir a contracorriente" de los fenómenos nacionales, conservando, sin embargo, una vitalidad del programa, pero con una movilidad de las organizaciones que es resultado y proyección de la crisis de la IV Internacional como partido internacional revolucionario?.

Durante estos años de contacto con el morenismo, Milcíades Peña colaborará con el trabajo teórico de la agrupación en donde milita. Escribirá artículos para la revista

\footnotetext{
${ }^{3}$ Coggiola, Ibíd., PP. 112-113.

${ }^{4}$ Coggiola, Ibíd., PP. 125-128.

${ }^{5}$ Camarero, op.cit., PP. 21-22.

${ }^{6}$ Camarero, Ibíd., pág. 29.

${ }^{7}$ Coggiola, op.cit., PP. 146-147.

${ }^{8}$ Tarcus Horacio, El marxismo olvidado en la Argentina: Silvio Frondizi y Milcíades Peña, Buenos Aires, Ediciones el Cielo por asalto. 1996. Pág. 354.

${ }^{9}$ Coggiola, op.cit., PP. 133-137.
} 
Estrategia de la Emancipación Nacional y participará en la redacción del libro La realidad argentina de Silvio Frondizi. En estos espacios el intelectual publicará sus posturas acerca del peronismo, la burguesía argentina y los estudios sobre el capitalismo y la industria argentina.

En estas publicaciones Peña expone por primera vez sus ideas acerca de la pseudoindustrialización. Dos artículos publicados en el primero y segundo tomo de Estrategia analizan el carácter del capitalismo argentino, la naturaleza de la burguesía y la oligarquía como clases dominantes, y el rol del peronismo en la industrialización argentina: uno es "Rasgos biográficos de la famosa burguesía industrial argentina", aparecido en 1956, y el segundo que lo complementa es "El imperialismo y la industrialización argentina", publicado en $1957^{10}$. Ya expondremos el trabajo de estas ideas en Fichas pero la síntesis de estas exposiciones es la siguiente:

- El atraso del capitalismo argentino se debe a su carácter correspondiente al de un país semicolonial condicionado (principalmente) por el imperialismo británico.

- En Argentina se desarrollaron determinadas industrias pero no hubo una industrialización sino una pseudoindustrialización.

- La burguesía argentina es "hermana de leche" de la oligarquía terrateniente y están relativamente unidas en sus intereses aunque estos no sean idénticos.

- El peronismo no revirtió la estructura de dominación de la oligarquía terrateniente y la burguesía industrial, ni sacó al capitalismo argentino de su estado de atraso por medio de un proceso de industrialización.

Siguiendo a Camarero en su estudio sobre la formación intelectual de Hermes Radio, se puede afirmar que estas ideas se encuentran primitivamente antes de que aparecieran en Estrategia. Recientemente ingresado al GOM-POR, Peña participa junto a Moreno en la elaboración de una serie de tesis para la formación militante donde se desarrollan las características del capitalismo argentino. Publicadas en Frente Proletario (periódico del POR) en 1948 aparecen las "Tesis industrial", la "Tesis agraria", "La acumulación primitiva de capital en la Argentina", las "Cuatro tesis sobre la colonización española y portuguesa" y la "Tesis Latinoamericana". En el mismo periódico, pero en 1951, aparecerá "La Argentina y el imperialismo",

Para los años 1958-1959 se dan los primeros conflictos teóricos con Palabra Obrera, señala Tarcus, como resultado de la política que desarrollaba el morenismo dentro del peronismo, pero también producto del cambio en la óptica de Peña respecto del peronismo y a algunos fenómenos latinoamericanos como la revolución boliviana y la cubana ${ }^{12}$.

\section{La revista Fichas de Investigación Económica y Social}

La segunda etapa de las relaciones de Peña con el morenismo corresponde al alejamiento y luego ruptura con Palabra Obrera en los últimos años de la década del '50.

\footnotetext{
${ }^{10}$ Tarcus, op.cit., PP. 318-324.

${ }^{11}$ Camarero, op.cit., pp 16-19.

12 Tarcus, op.cit., pág 353.
} 
Milcíades Peña deja de ser Hermes Radio a partir de este momento ${ }^{13}$. La tesis de Tarcus afirma que los giros del morenismo implicaban una presión para Peña que, siendo intelectual orgánico, debía ajustarse a la línea cambiante del partido según la coyuntura política $^{14}$. El intelectual pasó de ser antiperonista durante los gobiernos de Perón, mientras militaba en el GOM, POR y PSRN para luego estar del lado de los peronistas, en Palabra Obrera practicando "entrismo", pero sin dejar de ser antiperonista. Esto tendrá sus secuelas en los trabajos de Fichas cuando se publiquen investigaciones sobre el peronismo y el rol de la clase obrera. La vista aguda de Coggiola hace una crítica al respecto que luego retomaremos.

La participación de Peña en las publicaciones del "grupo Liberación” en 1959 será la expresión del alejamiento del autor de la corriente trotskista. Este sector es una escisión de Palabra Obrera que rechaza la política "entrista" de la agrupación y las caracterizaciones que hacía de la Revolución cubana como revolución burguesa ${ }^{15}$. Allí confluyen intelectuales como Peña y otros provenientes del MIR-Praxis de Silvio Frondizi, y sectores obreros con actividad sindical como Vitali y Speroni. Para Tarcus la señal de alejamiento del morenismo está en la tercera entrega de Revista de la Liberación. El artículo "16 tesis sobre Cuba" de Peña firmado con el pseudónimo de José Golan rebate las tesis de Moreno acerca de los sucesos en Cuba, los fenómenos nacionales y populistas, y las tareas de los marxista ante los mismos ${ }^{16}$.

Luego de la participación en el "grupo Liberación", Milcíades Peña se aboca en los próximos seis años, a la edición y publicación de Fichas de investigación económica y social que tendrá tiradas entre 1964-1966. Llegarán a ser diez números publicados donde participarán José Speroni, Jorge Schvarzer, Luis Franco, Halperín Donghi y Marcos Kaplan entre otros. La mayoría de los artículos escritos por Peña serán publicados con pseudónimos, en clave de polémica con la izquierda partidaria (tanto nacional como marxista) y con las ciencias sociales (en particular con la sociología heredera de Gino Germani), y con un estilo particular de análisis basado en una abultada cantidad de bibliografía y documentos consultados ${ }^{17}$.

Horacio González en el Prólogo a la edición facsimilar resalta el marxismo que se encuentra en Fichas, con un estilo de escritura influenciado por el mismo Marx, Wright Mills, Henri Lefebvre, Isaac Deutscher y León Trotsky. La obra también tiene una cierta "excepcionalidad" que por momentos es difícil encuadrarla en la objetividad que un análisis marxista de la realidad exige ${ }^{18}$. Luego retomaremos las críticas que Coggiola hace de este asunto, particularmente lo referido a la "excepcionalidad quietista" de la clase obrera peronista que Milcíades Peña analiza. Tomando textualmente de González la tesis maestra de la revista Fichas es que:

[...] el supuesto desarrollo industrial autónomo de la Argentina no existe, y que la "célebre burguesía industrial" que sostienen como hipótesis crucial los escritores de la

\footnotetext{
${ }^{13}$ Tarcus, Ibíd., pág. 359.

${ }^{14}$ Tarcus, Ibíd., pág. 117.

${ }^{15}$ Tarcus, Ibíd., pág. 354.

${ }^{16}$ Tarcus, Ibíd., PP. 361-365.

${ }^{17}$ González Horacio, "Fichas reescribir un 18 Brumario", Peña Milcíades (comp.), Fichas de Investigación económica y social. Volumen I, Edición facsimilar, Argentina, Ediciones Biblioteca Nacional, 2014, pp 7-9.

${ }^{18}$ González, Ibíd., pág. 12.
} 
izquierda nacional, no es más que una escisión interna de la clase terrateniente; y cuyo desarrollo es impulsado en el ámbito bien reconocible, a pesar de declaraciones y jactancias, de la política británica tradicional hacia el mercado interno argentino $[\ldots]^{19}$.

Ahora a analizaremos la idea de pseudoindustrialización que retoma el primer tomo de Fichas.

\section{Elementos para el análisis de la pseudoindustrialización argentina}

En el primer volumen de la revista Fichas aparecido en abril de 1964, Peña analiza una serie de cuestiones que llevan a comprender la realidad argentina desde la metodología y la teoría marxista. La primera cuestión es la estructura económica argentina de mediados de siglo XX entrelazada íntimamente con la segunda cuestión que es el carácter de la burguesía argentina. Estas dos grandes cuestiones son analizadas en dos artículos que organizan la primera publicación de la revista: "La evolución industrial argentina" y "La clase empresaria argentina". El primero artículo está a cargo de Víctor Testa, y para el segundo escriben Gustavo Polti y Hugo Berlatzky. Víctor Testa y Gustavo Polti son pseudónimos de Peña. En esta monografía utilizaremos solo el primer artículo, escrito por Víctor Testa.

Ambos artículos están planteados con la misma estructura. Primero establece "los hechos" y luego "las teorías". El análisis de la realidad y el relevamiento de los datos empíricos primero, y luego la explicación teórica y conceptual.

Los hechos en "La evolución industrial argentina" revelan entre 1937 y 1964 dos ciclos de la industria: crecimiento 1937-1943 y estancamiento 1947-1964. Lo importante que busca resaltar el análisis de los hechos, es el estancamiento de la evolución industrial argentina desde el fin de la II Guerra Mundial. Rebatiendo la idea de que el peronismo generó el "aluvión de nuevas industrias", Víctor Testa sostiene que el ascenso industrial se produjo con los gobiernos conservadores luego de la crisis del '29, y se mantuvo con políticas proteccionistas hasta 1947, mientras que en la etapa peronista la productividad decae, la industria tiende a una concentración mayor, la especialización de la producción es en torno a bienes de consumo no durables, y crecen los talleres manufactureros con escasa productividad. La evidencia está en los altos costos de producción, en la continua dependencia de importaciones de materias primas y maquinaria, y en la dependencia que caracteriza a la industria argentina respecto de las divisas que ingresen por la muralla aduanera $^{20}$. Este panorama también implica el decaimiento de la productividad por obrero a

\footnotetext{
${ }^{19}$ González, Ibíd., pp. 7-8.

20 Testa Víctor, "La evolución Industrial argentina", Peña Milcíades (comp.), Fichas de Investigación económica y social. Volumen I (abril 1964), Edición facsimilar, Argentina, Ediciones Biblioteca Nacional, 2014, PP. 44-45.
} 
pesar que el número de empleados aumenta (entre 1937-1960) y se triplica la maquinaria instalada. Maquinaria que Testa describe como importada y obsoleta, con dificultades de operar en el marco de servicios precarios en su funcionamiento (transporte y energía) y que lo único que genera es aumento en los costos de producción ${ }^{21}$.

La conducta de la burguesía en este contexto, dice Testa, es la misma que la de su pariente cercano, el terrateniente: obtener de manera parasitaria la máxima tasa de ganancia para el capital invertido. Porque la burguesía industrial argentina si bien es derrochadora, también debe reproducir su existencia ${ }^{22}$. Lo logra ampliando el número de maquinaria "obsoleta" (en el caso del terrateniente ampliando la superficie explotada) o explotando aún más al obrero ${ }^{23}$.

La explicación para esta situación no se reduce sólo a una actitud que pueda tener la burguesía, que depende de una circunstancia concreta, sino que Testa elabora un esquema explicativo que abarca la posición de Argentina en la división internacional del trabajo, las relaciones sociales de producción del país y las relaciones de propiedad.

El método utilizado por Testa para combinar estas aristas de una realidad es el del desarrollo desigual y combinado. Trotsky en Historia de la Revolución Rusa expone lo siguiente:

El desarrollo de una nación históricamente atrasada hace, forzosamente, que se confundan en ella, de una manera característica, las distintas fases del proceso histórico. Aquí el ciclo presenta, enfocado en su totalidad, un carácter confuso, embrollado, mixto.

[...] Azotados por el látigo de las necesidades materiales, los países atrasados vence obligados a avanzar a saltos. De esta ley universal del desarrollo desigual de la cultura se deriva otra que, a falta de nombre más adecuado, calificaremos de ley del desarrollo combinado, aludiendo a la aproximación de las distintas etapas del camino y a la confusión de distintas fases, a la amalgama de formas arcaicas y modernas. Sin acudir a esta ley, enfocada, naturalmente, en la integridad de su contenido material, sería imposible comprender la historia de Rusia ni la de ningún otro país de avance cultural rezagado, cualquiera que sea su grado ${ }^{24}$.

Este método permite a Testa llegar al nudo de la cuestión: la pseudoindustrialización es el reflejo del atraso argentino. Esta se diferencia de la industrialización propia de los países modelo del desarrollo capitalista: EEUU, Gran Bretaña y Francia, o de la industrialización "socialista" como la de la $\mathrm{URSS}^{25}$. Las características principales del proceso de industrialización son:

- Aumenta la composición técnica del capital, o sea la utilización de un número creciente de medios de producción más eficaces.

\footnotetext{
${ }^{21}$ Testa, Ibíd., PP. 57-58.

22 Testa menciona el "derroche" generado a través del gasto suntuario, la compra de extensiones de campo acentuando la existencia del latifundio, la instalación de maquinaria ineficiente, inversión en bolsas especulativas y depositando sus ganancias en bancos extranjeros como reaseguro ante una situación de crisis social (Testa, 45).

23 Testa, Ibíd., pág. 60.

${ }^{24}$ Trotsky León, Historia de la Revolución Rusa (1932), Buenos Aires, Ediciones ryr. 2012, PP. 30-31.

${ }^{25}$ Testa, op.ci., pág. 62.
} 
- Aumento en el desarrollo de industrias de medios de producción en comparación con aquellas industrias de medios de consumo.

- Tecnificación de la agricultura para sostener la demanda de la industria y de la fuerza de trabajo correspondiente a este sector.

- Movilidad mayor de recursos humanos y de materiales en la nación mediante un sistema de transporte amplio y eficaz.

- Modificaciones en la estructura de la sociedad, ante todo, modificaciones de las relaciones de propiedad; donde son expropiadas las viejas clases propietarias y ascienden nuevas clases al poder, fenómeno que revisten distintas manifestaciones políticas según las épocas y países, pero que en todos los casos acompañan la industrialización y sientan las bases para la misma. Las revoluciones democrático burguesas condensaron este proceso en las metrópolis imperialistas, subvirtiendo relaciones sociales pre capitalistas ${ }^{26}$.

La realidad contradictoria en la Argentina de mediados de siglo XX presenta un panorama distinto. La pseudoindustrialización existe debido a, por un lado, la presencia del imperialismo y al capital extranjero con un importante peso en la economía local haciendo del país una semicolonia, y por otro, a la presencia del latifundio en cuanto a las relaciones de propiedad. Así se da una parodia o caricatura de la industrialización ${ }^{27}$.

A principios de siglo XX Lenin planteaba en Imperialismo, fase superior del capitalismo que la semicolonia se caracteriza por ser país independiente políticamente, diferentes de las colonias -y por eso en una "situación transitoria"-, pero dependiente de los países imperialistas en sus redes de capital industrial y financiero principalmente ${ }^{28}$. El imperialismo, afirma Testa siguiendo a Lenin, perpetúa el atraso de la economía de la semicolonia, extrayendo de la misma la cuota de ganancia a través de la asfixia que genera el capital financiero. Mientras establece industrias en un medio atrasado, extrae del mismo los recursos naturales en calidad de materia prima, y financia la deuda del país por medio de $\operatorname{los}$ bancos $^{29}$. La burguesía terrateniente, como la llama Testa, y la burguesía industrial, en cuanto clases dominantes, tienen su interés principal en la cuota de ganancia como expusimos arriba, por lo que la perpetuación de la pseudoindustrialización es su principal modo de efectuarla sin entrar en conflictos con el imperialismo. Pero aquí entra otro factor que menciona Testa: el latifundio.

Con el latifundio la estructura de la propiedad agraria se mantiene en pocas manos: en las de los terratenientes. Pero no solo la propiedad, sino también la renta agraria que ésta genera mediante el sistema de arriendo. Para Testa esto implica una acción anti industrializadora, porque reduce el poder de consumo de la mano obra rural de aquellos bienes que circulan en el mercado interno, además de restarle ingresos a la nación que van a

\footnotetext{
${ }^{26}$ Testa, idem.

27 Testa, Ibíd., pág. 63.

${ }^{28}$ Lenin V.I., Imperialismo, fase superior del capitalismo (ensayo popular 1916), Pekín, Editorial del Pueblo, 1975, pp. 51-54.

${ }^{29}$ Lenin en El Imperialismo, fase superior del capitalismo caracteriza al imperialismo como: capitalismo monopolista con la producción concentrada, con las industrias organizadas en trust y cartels que buscan materias primas en el mercado mundial, con un desarrollo de las finanzas y bancos monopolizados por una oligarquía financiera, y con tendencia expansionista en la geografía mundial buscando colonias y semicolonias donde realizar la tasa de ganancia (Lenin, 83).
} 
parar en manos de los terratenientes ${ }^{30}$. También la renta agraria implica la especulación con el precio de la tierra por parte de los grupos que la concentran en sus manos, restándole posibilidad de agregarle valor mediante la intensificación y el mejoramiento técnico de la producción. En caso que estos existieran, menciona Testa, las ventajas obtenidas van a parar a las manos del terrateniente.

Estos factores -latifundio y dependencia del capital extranjero- generan los hechos que Testa observa en la realidad empírica, que ya fueron mencionados más arriba. Un desarrollo industrial serio para Testa (para Milcíades Peña en realidad) implica una salida política donde los explotados obreros y los chacareros del ámbito rural se hagan cargo del proceso que ni la burguesía argentina ni el peronismo realizaron mediante una revolución democrático burguesa. Las implicancias políticas que derivan de esto tienen sus raíces en posiciones que Trotsky plasmó en textos como la Revolución Permanente y el Programa de Transición. De estos textos Milcíades Peña toma dos grandes conclusiones que son parte del programa del marxismo para países atrasados y semicoloniales. Primero la idea de revolución permanente con tareas democráticas irresueltas por la burguesía nacional, donde obreros y campesinos realizan la expropiación de los medios de producción, y la reforma agraria a la vez. Dos de las Tesis fundamentales de la Revolución Permanente, exponen lo siguiente:

II Con respecto a los países de desarrollo burgués retrasado, y en particular de los coloniales y semicoloniales, la teoría de la revolución permanente significa que la resolución íntegra y efectiva de sus fines democráticos y de su emancipación nacional tan sólo puede concebirse por medio de la dictadura del proletariado, empuñando éste el poder como caudillo de la nación oprimida y, ante todo, de sus masas campesinas.

III El problema agrario, y con él el problema nacional, asignan a los campesinos, que constituye la mayoría aplastante de la población de los países atrasados, un puesto excepcional en la revolución democrática. Sin la alianza del proletariado con los campesinos, los fines de la revolución democrática no sólo no pueden realizarse, sino que ni siquiera cabe plantearlos seriamente. Sin embargo, la alianza de estas dos clases no es factible más que luchando irreconciliablemente contra la influencia de la burguesía liberal-nacional ${ }^{31}$.

Y segundo: la lucha por el socialismo en los países atrasados es a la vez una lucha por la liberación contra el imperialismo. Al respecto El Programa de transición plantea:

[...] la lucha por las más elementales reivindicaciones de independencia nacional y democracia burguesa se combina con el combate socialista contra el imperialismo mundial. En esta lucha las consignas democráticas, las reivindicaciones transitorias y los problemas de la revolución socialista no constituyen etapas históricas aisladas, sino que están íntimamente unidas. [...] La tarea central en los países coloniales y semicoloniales es la revolución agraria, es decir, la liquidación de la herencia feudal y la independencia nacional, es decir, la liberación del yugo capitalista. Ambas tarea están íntimamente ligadas ${ }^{32}$.

\footnotetext{
${ }^{30}$ Testa, op.cit., pág. 71.

31 Trotsky León, La Revolución Permanente (1930), Proyecto Espartaco 2000-2002, sin número.

32 Trotsky León, El programa de Transición (1938), edición online, pp. 18-19.
} 
Estos son los planteos fundamentales que centran el debate con Jorge Abelardo Ramos en Industria, Burguesía industrial y liberación nacional (aparecido en 1974). Debate que se encuentra en su totalidad en ese texto mencionado pero que Peña ya estaba desarrollando en las últimas apariciones de la revista Fichas.

Con este párrafo sintetizaremos la exposición de Testa y el contenido político de una industrialización que saque del atraso a la argentina de mediados de siglo XX:

Actualmente [principio de los años '60] el capital, extranjero o nacional, extrae sus ganancias más elevadas de la explotación del atraso de los países como Argentina y les cierra el camino hacia la industrialización, perpetuando su atraso mediante un desarrollo de tipo combinado. En última instancia, el capital constituye el límite último a la industrialización argentina, ya que el capital tiende necesariamente a una cuota máxima de ganancia y, en los países atrasados como el nuestro, la ganancia más elevada brota fundamentalmente de la explotación del atraso. Por ello, como lo atestigua toda la historia del siglo XX, sobre la base de la propiedad privada de los medios de producción los países atrasados no pueden dejar de serlo. Divorciado el capital del desarrollo de las fuerzas productivas, la industrialización de los países atrasados sólo es concebible en el marco de un sistema de producción interesado en liquidar el atraso, es decir, con una economía socialista ${ }^{33}$.

\section{Conclusiones}

El cierre del trabajo podemos plantearlo en torno a una serie de debates suscitados en torno a la obra y a la personalidad tanto militante como intelectual de Milcíades Peña.

La primera cuestión es acerca de la posibilidad de coexistencia que hubo en Peña entre el intelectual marxista que era y el partido donde trabajaba orgánicamente. Horacio Tarcus menciona que el intelectual revolucionario fue incompatible con el intelectual orgánico, que la teoría y la praxis entraron en crisis desde que Peña deja de estar bajo la línea partidaria de Moreno. Por otro lado Hernán Camarero también rescata este punto, acerca de las posibilidades de acción de un intelectual como era Peña dentro de un partido, y la idea del legado trotskista que Nahuel Moreno le imprime a Peña, pero lo plantea como una cuestión que debe ser problematizada en los estudios de la izquierda partidaria argentina. En ese sentido es cuestionable la postura de Tarcus (que mantiene en todo su trabajo, tanto para Peña como para Silvio Frondizi) acerca de la imposibilidad de ligazón entre teoría y praxis e intelectualidad y militancia que hay dentro de un partido marxista.

\footnotetext{
${ }^{33}$ Testa, op.cit., pág. 71. Aclaración entre corchetes es mía.
} 
Pero esto no es comprensible sin la contextualización del trotskismo argentino que debió enfrentarse a la contemporaneidad del peronismo el cual, como fenómeno histórico, aún hasta el día de hoy parte aguas en la política argentina. Había que explicar el fenómeno y definir tareas que el marxismo debía llevar a cabo con el movimiento obrero. Había que polemizar dentro de la izquierda sobre temas como la industrialización en la Argentina, el carácter del peronismo, el rol de las clases dominantes y de las clases explotadas. Peña mantuvo polémicas con Silvio Frondizi, con Rodolfo Puiggrós y al final de su vida con Abelardo Ramos. Luego de separarse del morenismo tuvo también su réplica con este. El problema surge, como plantea Osvaldo Coggiola, cuando "la excepción" y el "como sí" reemplazan la especificidad histórica de un fenómeno en el análisis teórico. Esto también lo observa Horacio González en su prólogo a la reedición de Fichas ${ }^{34}$. Tomaremos una cita textual de Coggiola para sintetizar la idea:

En 1958 "Hermes Radio" teorizaba en la revista Estrategia el "entrismo orgánico" de $P O$ [Palabra Obrera]: "la lucha democrática por la legalidad del partido [peronista] y del líder [Perón] que agrupan a la clase obrera, conduce directamente a la lucha socialista por el armamento del proletariado y la expropiación de la oligarquía". Si esto fuese cierto, se podía ahorrar la lucha por la organización y politización independiente del proletariado (o sea, el programa del trotskismo). Seis años después 1964- "Radio" Peña volvía de su ilusión, atribuyéndole la culpa a la clase obrera: "desde 1945 la clase obrera argentina, a nivel de actitudes y conducta, sobre todo de conducta, acepta el sistema social imperante... del conjunto de características que presenta la conducta de la clase obrera predominan, sobresalen, el quietismo, el conservadorismo... si en la Argentina la clase obrera desplegase una alta combatividad en procura de un objetivo puramente conservador, como sería la legalidad electoral para Perón, es probable que la clase se viera enfrentada a todo el sistema social ${ }^{35}$.

Para Milicíades Peña la clase obrera era conservadora por el hecho de no luchar contra Perón por la independencia política, y por las conquistas que consiguió al "mudarse" de las zonas rurales y atrasadas, a las urbanas e industriales. Dejó de ser revolucionaria para creer en su "Dios criollo": Perón. Coggiola señala que Peña justificó esto con un subjetivismo y oportunismo ("entrismo" mediante) que no terminaron de despegarse de las posturas del morenismo con respecto a la clase obrera ${ }^{36}$. Con respecto a las conclusiones sobre la burguesía nacional, el autor señala que el análisis de Peña es incompleto por su unilateralidad. No logró considerar objetivamente el aspecto progresivo del peronismo como fenómeno histórico de carácter nacionalista y burgués que si bien claudica ante el imperialismo (y en eso tenía razón Peña), también confronta con este ${ }^{37}$. Porque la burguesía

\footnotetext{
${ }^{34}$ González señala el "como si” de Peña que implican justificaciones objetivas a sus juicios de valor. La clase obrera es "como si" fuera revolucionaria, pero no lo era el 17 de Octubre del ' 45 porque claudicó ante los sindicalistas, los policías y el Ejército que organizaron la resistencia al encarcelamiento de Perón; la clase obrera es "como si" fuera móvil y revolucionaria pero no, porque era "quietista" y conservadora (González, $11)$.

${ }^{35}$ Coggiola, op.cit., pág. 161. Las aclaraciones entre corchetes son mías.

${ }^{36}$ Coggiola, ídem.

${ }^{37}$ Coggiola, Ibíd., PP. 177-178.
} 
de países coloniales y semicoloniales también está oprimida por la burguesía imperialista, y también tiene posiciones políticas independientes frente a la misma. Para Peña el peronismo era una continuación del orden conservador; una forma más de gobierno reaccionario, y esto no le permitió analizar las posibilidades reales de la clase obrera frente al mismo.

La principal falla estuvo en la herencia teórica y estratégica que adoptaba la organización morenista, primero el POR y luego PO, aún presentes en Peña luego de abrirse de la militancia orgánica. La otra falla estuvo en el alejamiento de Peña del movimiento real del proletariado y de los explotados desde el cual escribía los artículos de Fichas. Eso explica lo que Omar Acha denomina como descontento o desencanto del autor con las acciones de la clase obrera frente al peronismo ${ }^{38}$. Similar a la conclusión de Coggiola pero desde otro ángulo, Acha afirma que para Peña la clase obrera era el núcleo definitivo donde residía la resolución de la "tragedia de la historia argentina", pero que esta resolución terminó implicando más un anhelo subjetivo del autor que la lucha real por superar la historia trágica de nuestro país ${ }^{39}$.

El mérito de Milcíades Peña es haber llevado al máximo la crítica a los apologistas que sostenían el carácter revolucionario del peronismo en relación con las tareas que una revolución democrático burguesa resuelve (entre ella la lucha por la liberación nacional, la reforma agraria y la industrialización de la nación), mientras que, por otro lado, sentó las bases historiográficas para estudiar la historia argentina desde la perspectiva marxista.

Recibido: 18 agosto 2015

Aceptado: 20 octubre 2015

\section{Bibliografía}

Acha Omar, "Capítulo 6: Milcíades Peña y el proyecto de una historia trotskista" en Historia crítica de la historiografía argentina: las izquierdas en el siglo XX, Buenos Aires: Prometeo Libros. 2009. 249-299.

Camarero Hernán, "El período formativo de un intelectual: Milcíades Peña y el trotskismo en las décadas de 1940-1950". Archivos de Historia del movimiento y la izquierda, $\mathrm{N}^{\circ}$ 3, 2013. 9-33.

Coggiola Osvaldo, Parte I capítulos III y IV y Parte II capítulos IV y V, Historia del trotskismo en Argentina y América Latina, Buenos Aires: Ediciones ryr, 2006. 101-211 y 425-433.

González Horacio, "Fichas: reescribir un 18 Brumario", en Peña Milcíades (comp.), Fichas de Investigación económica y social. Volumen I. Edición Facsimilar, Argentina: Ediciones Biblioteca Nacional. 2014. 7-15.

\footnotetext{
${ }^{38}$ Acha Omar, Historia crítica de la historiografía argentina: las izquierdas en el siglo XX, Buenos Aires, Prometeo Libros, 2009, PP. 280-281.

${ }^{39}$ Acha, Ibíd., pág. 282.
} 
Lenin V. I., Imperialismo, fase superior del capitalismo (ensayo popular 1916). Pekín: Editorial del Pueblo. 1975.

Tarcus Horacio, "Introducción y capítulos 2, 4 y 5" en El marxismo olvidado en la Argentina: Silvio Frondizi y Milcíades Peña. Buenos Aires: Ediciones el Cielo por el asalto. 1996. 17-42, 65-120, $161-310$ y $311-413$.

Testa Víctor (pseudónimo Milcíades Peña), “1. La Evolución industrial Argentina”, en Milcíades Peña (comp.), Fichas de Investigación económica y social Tomo I (abril 1964), Volumen I. Edición Facsimilar. Argentina: Ediciones Biblioteca Nacional. 2014.

Trotsky León 1932, "Capítulo I Las características del desarrollo de Rusia", Historia de la Revolución Rusa. Buenos Aires: Ediciones ryr. 2012. 29-38.

Trotsky León 1930, “Qué es la Revolución Permanente? (Tesis fundamentales)”, La Revolución Permanente, Proyecto Espartaco 2000-2002.

Trotsky León 1938, "Los países atrasados y el programa de transición”, El Programa de Transición, edición online. 18-20. 